\title{
Mindfulness Based Therapy in Cancer Patients: Meta-Analysis
}

\section{Kamna Yadav', Samanta Puspak Kumar Jena ${ }^{2}$}

${ }^{1}$ Department of Psychology, North Campus, University of Delhi, Delhi 07, India. ${ }^{2}$ Department of Applied Psychology, South Campus, University of Delhi, Delhi 21, India.

\begin{abstract}
Evidence suggested that mindfulness-based techniques have been effective in increasing psychological well-being. The present meta-analytic study aimed to examine the effects of mindfulness-based programmes on anxiety, and depression in cancer infected and survivors. Improvement in mindfulness across groups were also studied. Using various search items (e.g., MBCT, MBSR, Cancer). A systematic search of the electronic databases, reference list, and journal content lists were conducted from June 2011 to June 2020. Fourteen randomized controlled trials $(\mathrm{N}=1831)$ evaluating effect of mindfulness in reducing anxiety and depression in cancer, measuring at pre-and-post interventions were included in this study. Random effects model was utilized for effect-size calculation. Interventions were between 5-8 weeks, with majority of participants been female (98.79\%). Significant medium effect was observed for anxiety $(\mathrm{g}=0.34)$ and depression $(\mathrm{g}=0.32)$ with moderate heterogeneity and higher for mindfulness $(g=1.061)$ with high heterogeneity. Publication bias was not observed. Mindfulness has substantial effects in reducing anxiety and depression in cancer infected and survivors.
\end{abstract}

Keywords: Mindfulness based cognitive therapy- mindfulness-based stress reduction- randomized controlled trial- cancer

Asian Pac J Cancer Care, 6 (3), 329-338

Submission Date: 05/13/2021 Acceptance Date: 07/26/2021

\section{Introduction}

Steady escalation of interest has been observed in studying effects of mindfulness on psychological, and physiological factors. Mindfulness has its roots in Buddhism focussing on open and receptive mode [1]. From more than a decade, Mindfulness Based Cognitive Therapy [2] and Mindfulness Based Stress Reduction Therapy [3] has become widely used psychological tool in oncology. Both have been developed for group settings but recently a web-based studies have been done, focussing on individual intervention $[4,5]$ and shown to be effective on psychological distress [6], fatigue [7], fear of recurrence [8], anxiety [9], depression [10, 11]. It helps in disengaging individuals from repetitive negative thought patterns to doing mode helping them to use mindful processing of experience [12]. It helps to be focused and aware about the present moment rather than ruminating about the past (regretting about life, ruminating about causes of cancer) and future worries (fear of death, fear of recurrence) to reduce sufferings [12].

Individuals suffering from life-threatening illnesses are at increased risk for developing psychological and emotional distress, further effecting overall health [13]. Stronger evidence for increased level of mental health problems has been reported in individuals suffering from cancer, HIV/AIDS, and asthma [14, 15]. Approximately $38 \%$ of individuals with cancer reported of suffering from depression [16] and 18\% from anxiety [17] and $35 \%$ of individuals reported of both anxiety and depression $[18,19]$. Further interfering to cope with illness leading to high level of distress adversely affecting progression and survival of cancer [20, 21].

Earlier meta-analytic study [22] reported moderate effect size for anxiety and mood symptoms (Hedge's $g$ $=0.63$, and 0.45 ) respectively. Decrease in depression (SMD: -0.37) and anxiety (SMD: -0.51), demonstrated evidence for the effectiveness of mindfulness but comprised a smaller number of RCTs leading to limited interpretability and generalizability of results [23]. In still another meta-analysis [24], showed moderate effect size of mindfulness on anxiety (Hedge's $g=0.37$ ) and depression (Hedge's $g=0.44$ ) but, included both Non-RCTs and RCTs thus, its interpretability is not limited to only RCTs.

\footnotetext{
Corresponding Author:

Dr. Kamna Yadav

PhD Scholar, Department of Psychology, North Campus, University of Delhi, Delhi 07, India.

Email: kamna.yadav89@gmail.com
} 
It further lacks use of stringent design (Randomization with active controls as comparison). Since, several RCTs has been conducted and literature for mindfulness in cancer has doubled, systematic reviews have been done but this is the first meta-analysis, which is conducted by using stringent design that includes RCTs with active controls as comparison to see the effect of mindfulness on anxiety and depression in cancer patients.

\section{Methods}

\section{Eligibility Criteria: Studies}

RCTs were included from June'2011- June'2020 providing MBCT or MBSR interventions as per Kabat-Zinn [3] and Segal, Williams, and Teasdale [25] guidelines. Both, offline and online studies with at least 5-8 weeks program were considered. Published studies which were translated and available in English were eligible. Thus, unpublished studies, non-randomized, dissertations, study-protocols, and abstracts were excluded. This has reduced potentially lower methodological quality and selective reporting bias.

\section{Types of Participants}

Participants of any age and across 0-IV stage with current diagnosis of cancer, receiving no psychological disorder treatment were considered. Those who reported of suffering from any clinical psychological disorder were excluded. Participants were either invited to participate or were self-referred at intervention set-up.

\section{Types of Interventions}

Studies were eligible if they had a pre-post design with an outcome standard measure of anxiety and depression, with reported data sufficient for estimating effect size, wherein data was available for both the groups. Studies were excluded if deviations from the standard program such as mindfulness art therapy, music therapy, shortened week structure, no home-work assignments were observed. Studies combining MBCT/MBSR with other type of interventions (e.g., yoga) without calculating MBCT/MBSR results separately were excluded.

\section{Types of Outcome Measures}

Outcome measures were anxiety and depression, measured on a standard instrument, administered at pre-post to both the groups. Mindfulness was measured on various instruments including, revised version of Cognitive and Affective Mindfulness Scale (CAMS-R) [26], Kentucky Inventory of Mindfulness Skills (KIMS) [27] and Five Facets of Mindfulness Questionnaire (FFMQ) [28]. Although these questionnaires were similar in measuring mindfulness, appear to be dissimilar in terms of number and construct of mindfulness [29]. As FFMQ measured five factors (acting with awareness, describing with words, noticing sensations, nonreactivity to inner experience, nonjudging of experience), CAMS-R measured four factors (attention, awareness, acceptance, and present focus) whereas KIMS measured four factors (observing, describing, acting with awareness, and accepting without judgment). Outcome measures were excluded if unobtainable, not well-defined and lack of sufficient data.

\section{Search Strategies}

Search was conducted using five electronic data bases namely, PubMed, Cochrane, SAGE Pub, EMBASE and Google Scholar. The data were searched for English language publication using the following terms 'mindfulness', 'cancer', 'mindfulness-based stress reduction', 'randomized controlled trial', 'mindfulness based cognitive therapy', terms from year June' 2011 to June'2020. Reference list of articles and reviews were also searched, and relevant studies were identified. Authors of the paper were also contacted through email and requested to share their research papers. Out of eight only three authors reverted with an access to their articles. Unpublished articles were excluded.

\section{Selection Process}

Selection was narrowed and studies were selected by title and list was made for all the studies done on cancer patients using Mindfulness intervention. Abstracts were reviewed from the list and relevant studies were retrieved in full form text and studies meeting eligibility criteria were selected for evaluation by meta-analysis.

\section{Data Extraction}

The studies were evaluated for the quality and content. The data was extracted using data extraction template based on minimum standards guidelines [30]. The data extraction sheet was developed to extract the data from the studies keeping the following points in mind: (1) Socio-demographic details of the participants including age, gender, type of cancer and stage of cancer, treatment, (2) intervention characteristics including type of mindfulness intervention (MBSR/MBCT), types of groups, number of sessions and participants in each group, results and limitations, (3) characteristics of outcome measures i.e., severity of depression, anxiety, and mindfulness.

\section{Quality Assessment}

Jadad scale [31] was used to assess methodological quality of studies (Table 1).

\section{Summary Measures}

Mean anxiety and depression differences between intervention and control group from baseline to post intervention were outcome measures. Based on study, mean at baseline and post-intervention for both the groups were utilized with confidence interval, $p$-value and standard -deviation for effect-size calculation.

\section{Statistical Analysis}

Analysis was done using Comprehensive MetaAnalysis Software Version-3 [32]. Effect size (ESs) was calculated using hedge's $g$ for continuous measure of mindfulness, depression, and anxiety, using random-effect model. Hedge's g is Cohen's d variation [33] and can be 
expressed as small (0.2), medium (0.5) and large (0.8) [34]. Random effect model was utilized for heterogeneity (using $\mathrm{Q}$ and $\mathrm{I}^{2}$ ) and forest plots.

\section{Risk of bias}

Publication bias was assessed using funnel plots of study ESs and fail-safe $\mathrm{N}$ test. Asymmetry of the funnel test indicates publication bias. Inverted funnel with ESs symmetrical distribution indicates absence of bias [35].

\section{Evidence Synthesis: Study Selection}

Preferred Reporting Items for Systematic Review and Meta-Analyses was utilized for flow diagram [36], fourteen RCTs fulfilling the inclusion criteria were selected for meta-analysis. Refer Figure 1 for illustration of selection procedure. Comprehensive literature search produced 879 citations after adjusting duplicates; title screening led to removal of 673 protocols with obvious reason of not meeting inclusion criteria. 206 abstracts were screened and 168 were removed: reason being no mindfulness-based interventions, no randomized controlled trials. Due to full-text unavailability, three studies were excluded. Full-text examination of 38 studies revealed that 25 studies did not meet criteria, for example, few of the studies measured depression but did not measured anxiety [37-39], some measured anxiety but did not measured depression [40-42], few studies did not mention the mean of both the groups at baseline and post-intervention [40, 43]. Reference list scanning led to identification of one study [44]. No duplicate of the fourteen eligible studies were identified.

\section{Study Characteristics: Participants}

Of fourteen, six studies were conducted in U.S., six in Europe and two in China $[45,46]$. Characteristics of studies are summarized in Table 2 and Table 3.

\section{Methods}

All RCTs were retrieved from June' 2011 to June' 2020 published in English. All participants received either MBSR $(\mathrm{K}=9)$ or MBCT $(\mathrm{K}=3)$ intervention offline except one study [47] for depression and anxiety symptoms. Twelve studies were two-armed randomized controlled trial, and two studies were three-armed controlled trial $[48,49]$. Data was taken for two groups i.e., Mindfulness group and control group from 3-armed controlled group trial studies. Out of all 14 studies, eight studies compared MBSR to Usual Care (UC), six compared MBSR to Wait-List controls offering delayed intervention. One study provided supportive counselling [50] and one study offered convention care including medication, normal living conditions such as oral care, safety, diet, self-protection [46].

\section{Participants}

Sample size of fourteen studies varied from 16 [50] to 299 [51, 52], yielding 1831 participants, of these 1806 were female participants. Of these 903 were in mindfulness intervention group and 928 in control group (Table 2). Six studies had only breast cancer diagnosed participants and remaining eight studies had mixed cancer site with stage 0 -III $(\mathrm{K}=13)$ and one study had participants with 0-IV stage [46]. The mean age of the participants was 51.26 years (range:16-80) and majority of participants were women diagnosed with breast cancer (84.7\%). All studies reported on time data with mean, median, standard deviation, or interval. Since diagnosis, the average mean time across studies was 18.5 months (range: 2weeks-24months). No difference was observed between treatment and control group on diagnosis and time. Among 14 randomized controlled studies, eight studies reported active chemotherapy, radiation (range: $35.7 \%-70.64 \%$ ), one study reported oral chemotherapy [50], active radioiodine therapy [45] and three studies had no active treatment $[47,53,54]$. At baseline no significant difference in terms of age, education, marital status, employment, cancer stage, cancer type, income, time since diagnosis between treatment and control group was observed.

Follow-up assessment was observed from baseline (minimum) to 6 months (maximum). Specifically, six-month follow up were seen in three studies $[53,55,56]$, five studies showed three-month follow-up [44, 46, 48, 51, 52], one- month follow-up was observed in Johns et al., 2015 and remaining five studies showed no follow-up [45, 47, 49, 50, 54].

\section{Interventions}

Interventions were based on Jon Kabat-Zinn (MBSR, 1992) and William, Teasdale \& Seagal (MBCT, 2002) guidelines/protocol. Length of interventions varied from 5 weeks [45], 6 weeks $[51,52,54]$ to 8 weeks $[44,46-50,53$, $55,56]$, except two studies provided 7 -week intervention $[45,57]$. Mindfulness involved weekly 1.5 hours [50], 2 to 2.5 hours [49] session with 6-days a week home practice assignment guided by audio files. One day 6-hour retreat at 6 weeks was observed [44]. Sessions included guided instructions, handouts, readings, guided audios, home assignments. Formal mindfulness practices include body-scan, yoga-based mindfulness, sitting meditation, breathing space, and didactic teachings. MBCT included formal mindfulness practices with cognitive based techniques such as psychoeducation, exercises targeting depression and anxiety including rumination and physical problem related to cancer [50]. Intervention was delivered by trained professional i.e., psychologist in MBCT with prior experience of 3 years to 9 years across studies. Intervention was provided offline i.e., face to face across studies, except one study wherein intervention was provided online [47].

\section{Outcomes}

Depression and anxiety measures used in studies to obtain ES can be seen from Table 2. Out of all studies, five studies measured mindfulness using either Cognitive \& Affective Mindfulness Scale-Revised [26] $(\mathrm{K}=1)$ [52], Kentucky Inventory of Mindfulness Skills [27] $(\mathrm{K}=1)$ [50] and Five-Facet Mindfulness Questionnaire [28] $(\mathrm{K}=3)[48,59,53]$. Effect size was calculated using difference in means and was not influenced by difference 
in mindfulness scales.

\section{Assessment of risk of bias}

Methodological quality varied across studies (Table 4).

\section{Results}

Essential data was available for all the fourteen RCTs eligible for estimating effect size for anxiety and depression pre-post intervention (Table 2). Only five studies measured mindfulness. Means and standard deviations at pre and post-test was provided for both groups across all the studies. Refer to Table 2. Estimated effect size (Hedge's g) and statistical significance, confidence interval, Z-value, and assigned random weight for outcome measures can be observed through Figure 2 and Figure 3, respectively.

\section{Synthesis of result}

\section{Effects on anxiety}

Analysis of all fourteen studies using random effects in comparison to control group shows decreased anxiety $(\mathrm{g}=0.346 ; \mathrm{CI}=95 \% ; \mathrm{p}=0.000)$, indicating medium effect. Heterogeneity was moderate $(\mathrm{Q}=31.199$; $\left.\mathrm{p}=0.003 ; \mathrm{I}^{2}=58.332\right)$ and outliner was not detected. Removal of pilot study $(\mathrm{N}=35)$ (Johns et al., 2015) increased the effect $(\mathrm{g}=0.360 ; \mathrm{CI}=95 \% ; \mathrm{p}=0.000)$ and higher heterogeneity level was observed $(\mathrm{Q}=30.470$; $\left.\mathrm{p}=0.002 ; \mathrm{I}^{2}=60.617\right)$, without outliners.

\section{Effects on depression}

In analysis of all fourteen RCTs, mindfulness group in comparison to control group, demonstrated medium effect size $(\mathrm{g}=0.327 ; \mathrm{CI}=95 \% ; \mathrm{p}=0.000)$, indicating

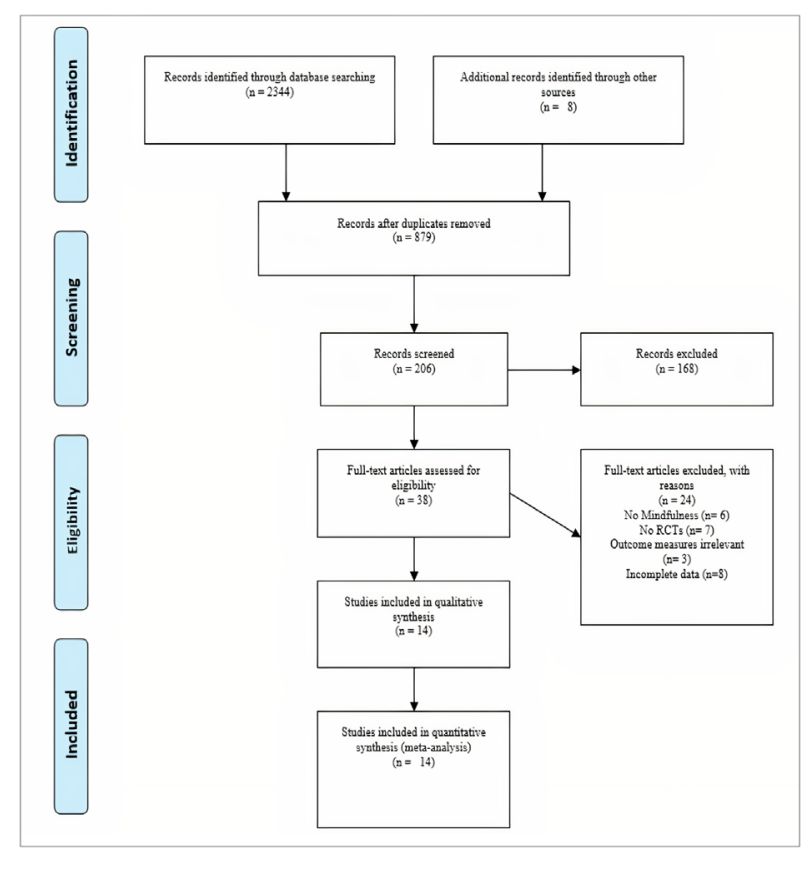

Figure 1. Study Selection Process via PRISMA Guidelines (2009)

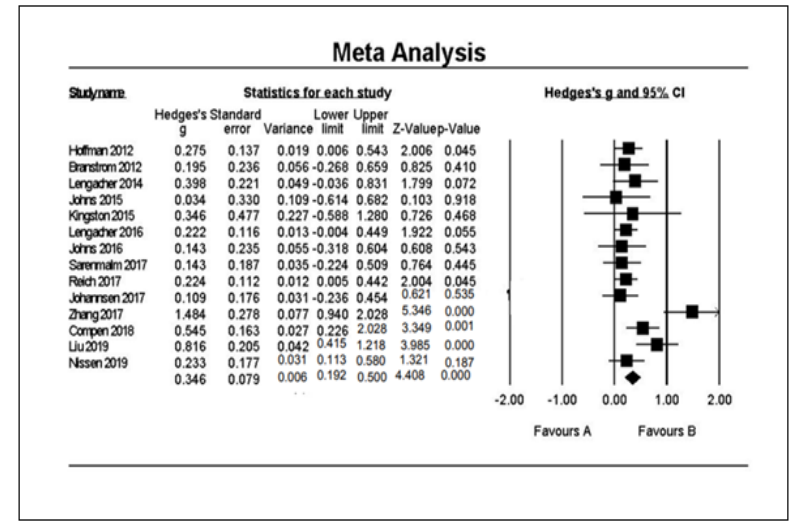

Figure 2. Forest Plot Demonstrating Intervention Effect on Anxiety: Pre-post between Group Effect.

reduced depression. Level of heterogeneity was moderate $\left(\mathrm{Q}=28.224 ; \mathrm{p}=0.008 ; \mathrm{I}^{2}=53.940\right)$, with no outliner. Removal of a study with small sample size $(\mathrm{N}=08)$ (Kingston et al., 2015) showed slightly increased effect size $(\mathrm{g}=0.330 ; \mathrm{CI}=95 \% ; \mathrm{p}=0.000)$ and increased heterogeneity $\left(\mathrm{Q}=28.217 ; \mathrm{p}=0.005 ; \mathrm{I}^{2}=57.472\right)$, without outliners.

\section{Effects on mindfulness}

In pooled data of five studies, treatment group in comparison to control group demonstrated a high effect in improving mindfulness ( $\mathrm{g}=1.061 ; \mathrm{CI}=95 \% ; \mathrm{p}=0.049)$ (Figure 4). With no outliner detected, level of heterogeneity was high $\left(\mathrm{Q}=133.684 ; \mathrm{p}=0.000 ; \mathrm{I}^{2}=97.008\right)$. Deletion of study Reich et al. (2016) showed higher effect size $(g=1.341 ; C I=95 \% ; p=0.082)$ with reduced heterogeneity level $\left(\mathrm{Q}=100.816 ; \mathrm{p}=0.000 ; \mathrm{I}^{2}=97.024\right)$.

\section{Risk of bias across studies}

Analysis demonstrated moderate to higher level of heterogeneity for intervention effects on outcome measures. High heterogeneity for mindfulness effects on intervention group was observed. To observe relation of heterogeneity to publication bias, demonstrating intervention effect on anxiety, and depression, funnel plot was developed. Johns et al. (2015) study was observed to made asymmetrical plot. Absence of publication bias was observed due to lower effect of afore mentioned study. Trim-and-fill method [35] demonstrated no point change of random effect model, showing no missing studies had changes in the effect size of the plot. Similar effects were observed in mindfulness effect distribution in comparison to anxiety and depression.

\section{Publication Bias}

Fail-safe $\mathrm{n}$ for depression and anxiety appeared to exceed the criteria for robustness of results. No evidence of asymmetry is observed for depression $\mathrm{t}(6.22), \mathrm{p}<0.000$ and anxiety t (6.78), $\mathrm{p}<0.000$ symptoms using Egger's regression test. 


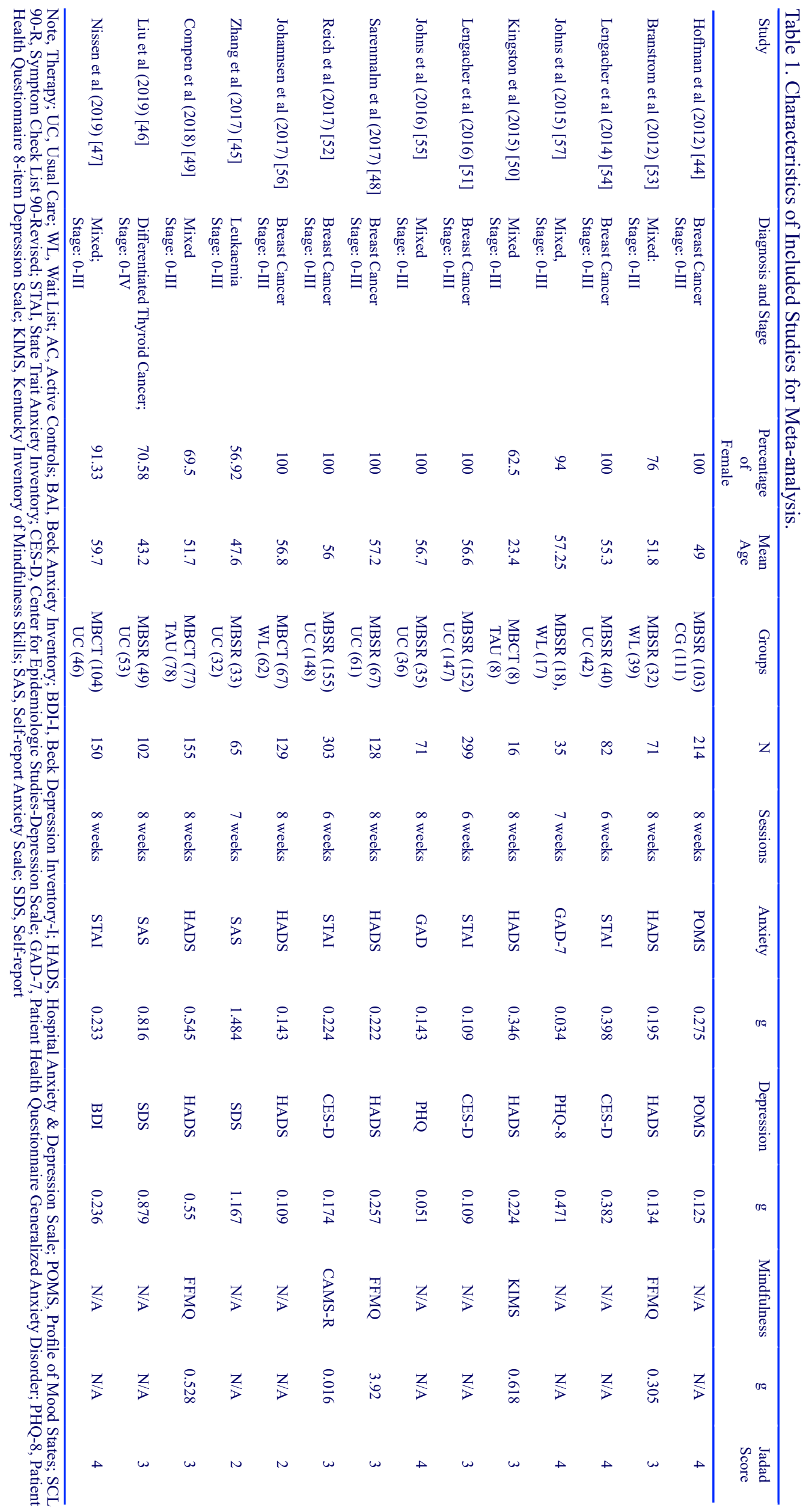


Table 2. Mean Comparison Across Variables in Included Studies (pre and post)

\begin{tabular}{|c|c|c|c|c|c|c|}
\hline \multirow[t]{2}{*}{ Study (Year) } & \multicolumn{3}{|c|}{ Anxiety scores } & \multicolumn{3}{|c|}{ Depression scores } \\
\hline & Tools & Pre-test & Post-test & Tools & Pre-test & Post-test \\
\hline \multirow[t]{2}{*}{ Hoffman et al. (2012) [44] } & POMS & $\mathrm{I}=13.16(7.2)$ & $\mathrm{I}=10.32(7.0)$ & POMS & $\mathrm{I}=12.79(10)$ & $\mathrm{I}=10(9.95)$ \\
\hline & & $\mathrm{C}=13.42(7.24)$ & $\mathrm{C}=13.36(7.20)$ & & $\mathrm{C}=15.7(12.79)$ & $\mathrm{C}=14.96(13.32)$ \\
\hline \multirow[t]{2}{*}{ Branstrom et al. (2012) [53] } & HADS & $\mathrm{I}=10.53(4.7)$ & $\mathrm{I}=8.32(4.40)$ & HADS & $\mathrm{I}=6.41(4.46)$ & $\mathrm{I}=4.85(4.20)$ \\
\hline & & $\mathrm{C}=10.44(4.59)$ & $\mathrm{C}=9.54(5.10)$ & & $\mathrm{C}=7.18(6.57)$ & $\mathrm{C}=6.57(4.04)$ \\
\hline \multirow[t]{2}{*}{ Lengacher et al. (2014) [54] } & STAI & $\mathrm{I}=35.3(12.0)$ & $\mathrm{I}=7.8(9.80)$ & CES-D & $\mathrm{I}=13.2(12.0)$ & $\mathrm{I}=7.2(4.60)$ \\
\hline & & $\mathrm{C}=40.4(11.9)$ & $\mathrm{C}=6.4(12.10)$ & & $\mathrm{C}=14.2(8.5)$ & $\mathrm{C}=4.0(1.70)$ \\
\hline \multirow[t]{2}{*}{ Johns et al. (2015) [57] } & GAD-2 & $\mathrm{I}=5.83(4.57)$ & $\mathrm{I}=3.91(4.24)$ & PHQ & $\mathrm{I}=7.89(5.41)$ & $\mathrm{I}=4.58(4.3)$ \\
\hline & & $\mathrm{C}=8.06(4.90)$ & $\mathrm{C}=5.92(4.18)$ & & $\mathrm{C}=8.94(5.17)$ & $\mathrm{C}=10.03(9.8)$ \\
\hline \multirow[t]{2}{*}{ Kingston et al. (2015) [50] } & HADS & $\mathrm{I}=8.4(5.50)$ & $\mathrm{I}=6.3(7.50)$ & HADS & $\mathrm{I}=8.0(6.2)$ & $\mathrm{I}=3.3(4.6)$ \\
\hline & & $\mathrm{C}=6.6(4.80)$ & $\mathrm{C}=7.5(5.10)$ & & $\mathrm{C}=8.5(6.4)$ & $\mathrm{C}=6.0(6.8)$ \\
\hline \multirow[t]{2}{*}{ Lengacher et al. (2016) [51] } & STAI & $\mathrm{I}=16.5(6.70)$ & $\mathrm{I}=15.7(5.10)$ & CES-D & $\mathrm{I}=16.5(6.7)$ & $\mathrm{I}=15.7(5.10)$ \\
\hline & & $\mathrm{C}=18.1(7.50)$ & $\mathrm{C}=18.3(6.40)$ & & $\mathrm{C}=18.1(7.5)$ & $\mathrm{C}=18.3(6.40)$ \\
\hline \multirow[t]{2}{*}{ Johns et al. (2016) [55] } & GAD-2 & $\mathrm{I}=7.47(5.50)$ & $\mathrm{I}=3.21(3.76)$ & PHQ-8 & $\mathrm{I}=11.35(5.57)$ & $\mathrm{I}=6.27(3.90)$ \\
\hline & & $\mathrm{C}=8.57(5.31)$ & $\mathrm{C}=5.28(4.27)$ & & $\mathrm{C}=12.53(4.90)$ & $\mathrm{C}=7.80(4.67)$ \\
\hline \multirow[t]{2}{*}{ Sarenmalm et al. (2017) [48] } & HADS & $\mathrm{I}=6.5(4.30)$ & $\mathrm{I}=6.0(3.90)$ & HADS & $\mathrm{I}=4.30(3.70)$ & $\mathrm{I}=3.30(3.30)$ \\
\hline & & $\mathrm{C}=4.8(3.60)$ & $\mathrm{C}=5.1(3.90)$ & & $\mathrm{C}=3.50(3.50)$ & $\mathrm{C}=3.80(3.80)$ \\
\hline \multirow[t]{2}{*}{ Reich et al. (2017) [52] } & STAI & $\mathrm{I}=38.62(12.3)$ & $\mathrm{I}=30.62(12.3)$ & CES-D & $\mathrm{I}=10.87(6.89)$ & $\mathrm{I}=8.12(5.45)$ \\
\hline & & $\mathrm{C}=35.86(11.29)$ & $\mathrm{C}=31.76(13.2)$ & & $C=10.04(6.46)$ & $\mathrm{C}=8.82(6.02)$ \\
\hline \multirow[t]{2}{*}{ Johannsen et al. (2017) [56] } & HADS & $\mathrm{I}=16.5(6.7)$ & $\mathrm{I}=15.7(5.1)$ & HADS & $\mathrm{I}=16.5(6.7)$ & $\mathrm{I}=15.7(5.1)$ \\
\hline & & $\mathrm{C}=18.1(7.5)$ & $\mathrm{C}=18.3(6.4)$ & & $\mathrm{C}=18.1(7.5)$ & $\mathrm{C}=18.3(6.4)$ \\
\hline \multirow[t]{2}{*}{ Zhang et al. (2017) [45] } & SAS & $\mathrm{I}=56.83(7.79)$ & $\mathrm{I}=40.91(10.03)$ & SDS & $\mathrm{I}=63.35(8.61)$ & $\mathrm{I}=46.87(7.48)$ \\
\hline & & $\mathrm{C}=56.91(8.11)$ & $\mathrm{C}=59.74(9.35)$ & & $\mathrm{C}=61.87(8.95)$ & $\mathrm{C}=58.93(7.43)$ \\
\hline \multirow[t]{2}{*}{ Compen et al. (2018) [49] } & POMS & $\mathrm{I}=18.81(6.7)$ & $\mathrm{I}=13.25(6.33)$ & POMS & $\mathrm{I}=18.81(6.7)$ & $\mathrm{I}=13.25(6.33)$ \\
\hline & & $\mathrm{C}=17.04(5.79)$ & $\mathrm{C}=16.37(6.50)$ & & $\mathrm{C}=17.04(5.79)$ & $C=16.37(6.5)$ \\
\hline \multirow[t]{2}{*}{ Liu et al. (2019) [46] } & SAS & $\mathrm{I}=59.58(9.62)$ & $\mathrm{I}=65.24(11.08)$ & SDS & $\mathrm{I}=53.60(8.61)$ & $\mathrm{I}=60.52(9.73)$ \\
\hline & & $\mathrm{C}=58.72(8.65)$ & $\mathrm{C}=77.37(14.5)$ & & $\mathrm{C}=54.36(9.81)$ & $\mathrm{C}=73.64(11.22)$ \\
\hline \multirow[t]{2}{*}{ Nissen et al. (2019) [47] } & STAI & $\mathrm{I}=41.7(10.2)$ & $\mathrm{I}=37.5(8.9)$ & BDI-II & $\mathrm{I}=15.1(7.4)$ & $\mathrm{I}=10.7(6.6)$ \\
\hline & & $\mathrm{C}=42.7(9.8)$ & $\mathrm{C}=41.8(11.9)$ & & $\mathrm{C}=17.6(7.5)$ & $\mathrm{C}=15.6(9.2)$ \\
\hline
\end{tabular}

Note, I, Intervention Group; C, Control Group; POMS, Profile of Mood States; HADS, Hospital Anxiety Depression Scale; STAI, State Trait Anxiety Inventory; GAD-2, Generalized Anxiety Disorder-2; SAS, Self-report Anxiety Scale; SDS, Self-report Depression Scale; CES-D, Center for Epidemiology Studies-Depression Scale; BDI-II, Beck Depression Inventory-II; PHQ, Patient Health Questionnaire-8 item depression scale

\section{Discussion}

Analysis outlines the effect of mindfulness on reduction of anxiety and depression and increment in mindfulness in cancer-infected and survivors with control group after intervention. Literature search led to fourteen RCTs conducted for reducing anxiety, depression and improved psychological well-being. Participants of all included studies were predominately females with diagnosis of breast cancer.

Mindfulness has become popular intervention amongst cancer individuals, but very little is known about its efficacy. Previously done meta-analysis [22] does not emphasised on mindfulness to decrease anxiety and depression in cancer infected and survivors but included differential population or effects on mental health and psychological problems [58]. Number of studies has been doubled in a brief amount of time, further, allowing for a more comprehensive meta-analysis to explore the effects of MBCT/MBSR for cancer patients particularly for RCTs.
Pooled analysis results suggested ES ranging from low to moderate range for reduction of depression (Hedge's $g=0.32$ ) and anxiety (Hedge's $g=0.36$ ) indicating robust and significant effect. Heterogeneity for studies was found out to be moderate for depression $(53.940, \mathrm{p}<0.008)$ and medium for anxiety (58.332, $\mathrm{p}<0.003)$ within and between studies and high for mindfulness $(97.008, \mathrm{p}<0.000)$. Quality result for all the studies were found out to be 3.2 suggesting reliable results.

Piet, Wurtzen and Zachariae (2012) represents systematic review of mindfulness, allowing for comparison with findings from present study. There RCTs findings matched our findings for anxiety and depression symptoms. Hoffman et al (2010) in their meta-analysis included wide range of medical and psychological disorders, they also included number of subgroup analyses showing pre-post effect size for anxiety $(\mathrm{g}=0.63)$ and $(0.45)$ and depression $(g=0.45$, respectively). These were based on few studies.

Mindfulness has been effective in decreasing 
Table 3. Mean Comparison of Mindfulness Across Groups (pre and post)

\begin{tabular}{lccc}
\hline Study (Year) & & Mindfulness Scores & \\
& Tool & Pre-test & Post-test \\
\hline Branstrom et al. (2012) [53] & FFMQ & $\mathrm{I}=123.45(32.74)$ & $\mathrm{I}=140.17(29.02)$ \\
& & $\mathrm{C}=117.65(32.69)$ & $\mathrm{C}=120.53(32.57)$ \\
Kingston et al. (2015) [50] & KIMS & $\mathrm{I}=118.1(17.4)$ & $\mathrm{I}=124.4(13.6)$ \\
& & $\mathrm{C}=123.9(15.8)$ & $\mathrm{C}=117.0(9.3)$ \\
Sarenmalm et al. (2017) [48] & FFMQ & $\mathrm{I}=16.4(3.7)$ & $\mathrm{I}=17.1(3.0)$ \\
& & $\mathrm{C}=17.1(3.5)$ & $\mathrm{C}=17.1(3.3)$ \\
Reich et al. (2017) [52] & CAMS-R & $\mathrm{I}=34.04(6.61)$ & $\mathrm{I}=35.20(7.55)$ \\
& & $\mathrm{C}=35.37(7.17)$ & $\mathrm{C}=36.71(7.52)$ \\
Compen et al. (2018) [49] & FFMQ & $\mathrm{I}=72.43(9.69)$ & $\mathrm{I}=82.02(10.42)$ \\
& & $\mathrm{C}=75.75(11.18)$ & $\mathrm{C}=77.26(11.80)$ \\
\hline
\end{tabular}

Note, I, Intervention Group; C, Control Group; CAMS-R, Cognitive and Affective Mindfulness Scale-Revised; FFMQ, Five-facet of Mindfulness Scale; KIMS, Kentucky Inventory of Mindfulness Skills

depression and anxiety symptoms, by targeting rumination and emotional avoidance, maintaining processes across anxiety and depression [59]. It is cost-effective treatment, aimed at improving emotion regulation through increased attention and acceptance. Mindfulness therapy can be used along with other interventions, focusing on change through active problem solving.

\section{Strengths}

First study investigating only RCTs to see the effect of Mindfulness (MBSR/MBCT) on anxiety and depression symptoms in cancer infected and survivors. Study aims, inclusion criteria, method of analysis were prespecified and highly focussed. Random-effects model was used [34].

\section{Limitations}

This meta-analysis is limited by basic statistical assumptions and inclusion criteria. Majority of individuals were diagnosed with breast cancer but differ with cancer stage and time since diagnosis, both between and within studies. Effects of mindfulness for different individual makes it difficult to come at a conclusion. It cannot be generalized to male cancer individuals as majority of participants were female with breast cancer.

\section{Implications for practice}

Evidence suggests that mindfulness interventions are delivered effectively in cancer patients $[45,46,53]$ and survivors $[47,55]$. Previous meta-analysis included studies on cancer survivors [23], non-RCTs [22, 24], studied either effect on depression [60], this is the first meta-analysis including RCTs of adult cancer infected i.e., patients on active treatment such as chemotherapy [45, 48, 49, 52, 56], radio-active iodine therapy [46]. Finding indicates that mindfulness intervention for cancer patients and survivors substantially decrease anxiety and depression and improved mindfulness. Given access to cancer patients, especially during active-treatment, mindfulness can be

Table 4. Assessment of Risk of Bias.

\begin{tabular}{|c|c|c|c|c|c|}
\hline Study (Year) & Blinding & $\begin{array}{c}\text { Allocation } \\
\text { Concealment }\end{array}$ & $\begin{array}{l}\text { Comparison of Groups on } \\
\text { outcome measure at baseline }\end{array}$ & $\begin{array}{c}\text { Dropouts \& withdrawal } \\
\text { details }\end{array}$ & $\begin{array}{c}\text { Intent to } \\
\text { treat } \\
\text { analysis }\end{array}$ \\
\hline Hoffman et al. (2012) [44] & Yes & Yes & Yes & Yes & Yes \\
\hline Branstrom et al. (2012) [53] & No & Yes & Yes & No & Yes \\
\hline Lengacher et al. (2014) [54] & Yes & Yes & Yes & Yes & Yes \\
\hline Johns et al. (2015) [57] & Yes & Yes & Yes & Yes & Yes \\
\hline Kingston et al. (2015) [50] & No & Yes & Yes & Yes & Yes \\
\hline Lengacher et al. (2016) [51] & No & Yes & Yes & Yes & Yes \\
\hline Johns et al. (2016) [55] & Yes & Yes & Yes & Yes & Yes \\
\hline Sarenmalm et al. (2017) [48] & No & Yes & Yes & No & Yes \\
\hline Reich et al. (2017) [52] & No & No & Yes & No & Yes \\
\hline Johannsen et al. (2017) [56] & No & No & Yes & Yes & Yes \\
\hline Zhang et al. (2017) [45] & No & No & Yes & No & Yes \\
\hline Compen et al. (2018) [49] & No & Yes & Yes & Yes & Yes \\
\hline Liu et al. (2019) [46] & No & Yes & Yes & No & Yes \\
\hline Nissen et al. (2019) [47] & Yes & Yes & Yes & Yes & Yes \\
\hline
\end{tabular}

Note, Adapted from Higgins and Altman (2008). 




Figure 3. Forest Plot Demonstrating Intervention Effect on Depression: Pre-post between Group Effect.

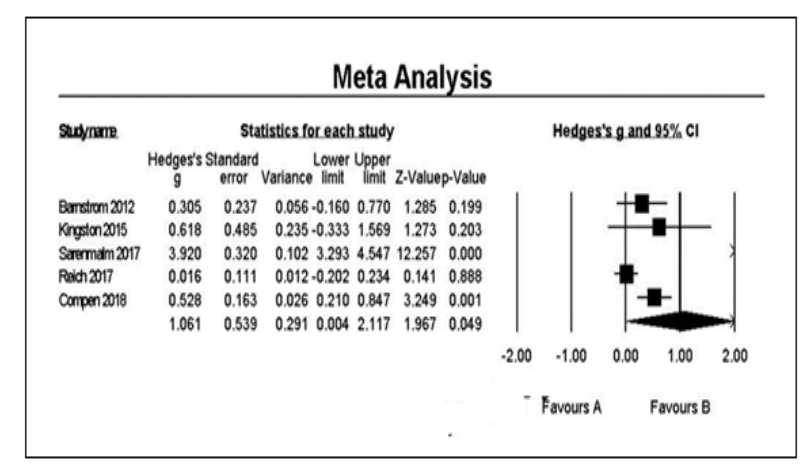

Figure 4. Forest Plot for Mindfulness: Pre-post.

effective compared to no psychological interventions.

\section{Implications for research}

Areas for future RCTs in cancer patients using mindfulness was identified: 1) comparison of face to face with internet-based mindfulness interventions with same content, involving a control group, 2) assessing home-based practices of mindfulness interventions as it is an integral part of the intervention, 3) assessing interventions effect on subgroups when possible and applicable e.g., by gender, income, age, occupation, and education, 4) since very few studies measured the increment of mindfulness at pre-post and even at follow-up, this can be future direction for research. Methodological quality characteristics including blinding, treatment adherence or non-adherence shall be reported by the researchers. Feedbacks from participants in research should be included. There are very few studies on effect of mindfulness on young adolescence (cancer) psychological well-being, considering it as need of an hour, warrants investigation.

Supplementary data to this article can be found online.

\section{Declaration of Conflicting Interests}

Author(s) declare that there is no financial, authorship, publication conflict of interest of this article. Funding

This research did not receive any specific grant from funding agencies in the public, commercial, or not-forprofit sectors.

\section{References}

1. Brown KW, Ryan RM. The benefits of being present: Mindfulness and its role in psychological well-being. Journal of Personality and Social Psychology. 2003;84(4):822-848. https://doi.org/10.1037/0022-3514.84.4.822

2. Segal Z, Williams J, Teasdale J. Mindfulness-based cognitive therapy for depression. New York: Guildford Press. 2012.

3. Kabat-Zinn J. Full catastrophe living: Using the wisdom of your mind and body to face stress, pain, and illness. New York: Delacorte. 1990.

4. Bruggeman Everts FZ, van der Lee ML, de Jager Meezenbroek E. Web-based individual Mindfulness-Based Cognitive Therapy for cancer-related fatigue - A pilot study. Internet Interventions. 2015 05;2(2):200-213. https://doi. org/10.1016/j.invent.2015.03.004

5. Compen FR, Bisseling EM, Van der Lee ML, Adang EMM, Donders ART, Speckens AEM. Study protocol of a multicenter randomized controlled trial comparing the effectiveness of group and individual internet-based Mindfulness-Based Cognitive Therapy with treatment as usual in reducing psychological distress in cancer patients: the BeMind study. BMC Psychology. 201508 13;3(1). https://doi.org/10.1186/s40359-015-0084-1

6. Sharplin GR, Jones SBW, Hancock B, Knott VE, Bowden JA, Whitford HS. Mindfulness-based cognitive therapy: an efficacious community-based group intervention for depression and anxiety in a sample of cancer patients. Medical Journal of Australia. 2010 09;193(S5). https://doi. org/10.5694/j.1326-5377.2010.tb03934.x

7. Labelle LE, Campbell TS, Faris P, Carlson LE. Mediators of Mindfulness-Based Stress Reduction (MBSR): Assessing the Timing and Sequence of Change in Cancer Patients. Journal of Clinical Psychology. 201408 05;71(1):21-40. https://doi. org/10.1002/jclp.22117

8. Lengacher CA, Reich RR, Post-White J, Moscoso M, Shelton MM, Barta M, Le N, Budhrani P. Mindfulness based stress reduction in post-treatment breast cancer patients: an examination of symptoms and symptom clusters. Journal of Behavioral Medicine. 201104 20;35(1):86-94. https:// doi.org/10.1007/s10865-011-9346-4

9. Wong SYS, Yip BHK, Mak WWS, Mercer S, Cheung EYL, Ling CYM, Lui WWS, Tang WK, Lo HHM, Wu JCY, Lee TMC, Gao T, Griffiths SM, Chan PHS, Ma HSW. Mindfulness-based cognitive therapy v. group psychoeducation for people with generalised anxiety disorder: Randomised controlled trial. British Journal of Psychiatry. 2016 07;209(1):68-75. https://doi.org/10.1192/ bjp.bp.115.166124

10. Ma SH, Teasdale JD. Mindfulness-Based Cognitive Therapy for Depression: Replication and Exploration of Differential Relapse Prevention Effects. Journal of Consulting and Clinical Psychology. 2004;72(1):31-40. https://doi. org/10.1037/0022-006x.72.1.31

11. Strauss C, Cavanagh K, Oliver A, Pettman D. MindfulnessBased Interventions for People Diagnosed with a Current Episode of an Anxiety or Depressive Disorder: A MetaAnalysis of Randomised Controlled Trials. Laks J. PLoS ONE. 201404 24;9(4):e96110. https://doi.org/10.1371/ journal.pone.0096110

12. Baer RA. Mindfulness training as a clinical intervention: A conceptual and empirical review. Clinical Psychology: Science and Practice. 2003;10(2):125-143. https://doi. 
org/10.1093/clipsy.bpg015

13. Naylor C, Parsonage M, McDaid D, Knapp M, Fossey M, Galea A. Long-term conditions and mental health: The cost of co-morbidities. 2012. 2012:1-32.

14. Chapman D, Perry G, Strine T. The vital link between chronic disease and depressive disorders. Preventive Chronic Disorder. 2005;2(1).

15. Sederer L, Silver L, McVeigh K, Levy J. Integrating care for medical and mental illnesses. Preventing Chronic Disease, Public Health Research. Practice and Policy. 2006;3(2):1-3.

16. Massie MJ. Prevalence of Depression in Patients With Cancer. Journal of the National Cancer Institute Monographs. 200407 01;2004(32):57-71. https://doi.org/10.1093/ jncimonographs/lgh014

17. Stark D, Kiely M, Smith A, Velikova G, House A, Selby P. Anxiety Disorders in Cancer Patients: Their Nature, Associations, and Relation to Quality of Life. Journal of Clinical Oncology. 200207 15;20(14):3137-3148. https:// doi.org/10.1200/jco.2002.08.549

18. Zabora J, BrintzenhofeSzoc K, Curbow B, Hooker C, Piantadosi S. The prevalence of psychological distress by cancer site. Psycho-Oncology. 2001 01;10(1):19-28. https://doi.org/10.1002/1099-1611(200101/02)10:1<19::aidpon501>3.0.co;2-6

19. Carlson LE, Angen M, Cullum J, Goodey E, Koopmans J, Lamont L, MacRae JH, Martin M, Pelletier G, Robinson J, Simpson JSA, Speca M, Tillotson L, Bultz BD. High levels of untreated distress and fatigue in cancer patients. British Journal of Cancer. 200405 25;90(12):2297-2304. https:// doi.org/10.1038/sj.bjc.6601887

20. Brown KW, Levy AR, Rosberger Z, Edgar L. Psychological Distress and Cancer Survival. Psychosomatic Medicine. 2003 07;65(4):636-643. https://doi.org/10.1097/01. psy.0000077503.96903.a6

21. Lutgendorf SK, Sood AK. Biobehavioral Factors and Cancer Progression. Psychosomatic Medicine. 2011 Nov;73(9):724730. https://doi.org/10.1097/psy.0b013e318235be76

22. Hofmann SG, Sawyer AT, Witt AA, Oh D. The effect of mindfulness-based therapy on anxiety and depression: A meta-analytic review.. Journal of Consulting and Clinical Psychology. 2010;78(2):169-183. https://doi.org/10.1037/ a0018555

23. Cramer H, Lauche R, Paul A, Dobos G. MindfulnessBased Stress Reduction for Breast Cancer-A Systematic Review and Meta-Analysis. Current Oncology. 2012 Oct 01;19(5):343-352. https://doi.org/10.3747/co.19.1016

24. Piet J, Würtzen H, Zachariae R. The effect of mindfulnessbased therapy on symptoms of anxiety and depression in adult cancer patients and survivors: A systematic review and meta-analysis. Journal of Consulting and Clinical Psychology. 2012 Dec;80(6):1007-1020. https://doi. org/10.1037/a0028329

25. Segal Z, Williams J, Teasdale J. Mindfulness based cognitive therapy for depression: A new approach to preventing relapse. New York: Guildford Press. 2002.

26. Feldman G, Hayes A, Kumar S, Greeson J, Laurenceau J. Mindfulness and Emotion Regulation: The Development and Initial Validation of the Cognitive and Affective Mindfulness Scale-Revised (CAMS-R). Journal of Psychopathology and Behavioral Assessment. 2006 Nov 07;29(3):177-190. https:// doi.org/10.1007/s10862-006-9035-8

27. Baer RA, Smith GT, Allen KB. Assessment of Mindfulness by Self-Report. Assessment. 2004 09;11(3):191-206. https:// doi.org/10.1177/1073191104268029

28. Baer RA, Smith GT, Hopkins J, Krietemeyer J, Toney L. Using Self-Report Assessment Methods to Explore Facets of Mindfulness. Assessment. 2006 03;13(1):27-45. https:// doi.org/10.1177/1073191105283504

29. Sauer S, Walach H, Schmidt S, Hinterberger T, Lynch S, Bussing A, Kohls N. Assessment of mindfulness: review on state of the art. Mindfulness. 2013;4(1):3-17.

30. Reviews C. Cochrane Consumers and Communication Review Group, Data Extraction Template for Included Studies London. In: Collaboration TC, editor. UK2015.

31. Jadad AR, Moore R, Carroll D, Jenkinson C, Reynolds DM, Gavaghan DJ, McQuay HJ. Assessing the quality of reports of randomized clinical trials: Is blinding necessary? Controlled Clinical Trials. 1996 02;17(1):1-12. https://doi. org/10.1016/0197-2456(95)00134-4

32. Borenstein M, Hedges L, Higgins J, Rothstein H. Comprehensive Meta-Analysis (CMA) 3ed. Englewood, NJ: Biostat; 2016. p. Computer Program.

33. J. C. Statistical Power Analysis for the Behavioral Sciences. New York Lawrence Erelbaum Associates 1988.

34. Hedges LV, Vevea JL. Fixed- and random-effects models in meta-analysis. Psychological Methods. 1998;3(4):486-504. https://doi.org/10.1037/1082-989x.3.4.486

35. Rothstein H, Sutton A, Borenstein M. Publication Bias in Meta-analysis. In: Rothstein HR SA, Borenstein M. , editor. Publication Bias in Meta-Analysis: Prevention, Assessment and Adjustments UK: Wiley 2005.

36. Moher D, Liberati A, Tetzlaff J, Altman DG. Preferred Reporting Items for Systematic Reviews and MetaAnalyses: The PRISMA Statement. PLoS Medicine. 2009 07 21;6(7):e1000097. https://doi.org/10.1371/journal. pmed. 1000097

37. Garland SN, Carlson LE, Stephens AJ, Antle MC, Samuels C, Campbell TS. Mindfulness-Based Stress Reduction Compared With Cognitive Behavioral Therapy for the Treatment of Insomnia Comorbid With Cancer: A Randomized, Partially Blinded, Noninferiority Trial. Journal of Clinical Oncology. 201402 10;32(5):449-457. https://doi. org/10.1200/jco.2012.47.7265

38. Boyle CC, Stanton AL, Ganz PA, Crespi CM, Bower JE. Improvements in emotion regulation following mindfulness meditation: Effects on depressive symptoms and perceived stress in younger breast cancer survivors.. Journal of Consulting and Clinical Psychology. 2017 04;85(4):397-402. https://doi.org/10.1037/ccp0000186

39. Witek Janusek L, Tell D, Mathews HL. Mindfulness based stress reduction provides psychological benefit and restores immune function of women newly diagnosed with breast cancer: A randomized trial with active control. Brain, Behavior, and Immunity. 2019 08;80:358-373. https://doi. org/10.1016/j.bbi.2019.04.012

40. Henderson VP, Clemow L, Massion AO, Hurley TG, Druker S, Hébert JR. The effects of mindfulness-based stress reduction on psychosocial outcomes and quality of life in early-stage breast cancer patients: a randomized trial. Breast Cancer Research and Treatment. 201109 08;131(1):99-109. https://doi.org/10.1007/s10549-011-1738-1

41. Blaes A, Fenner D, Bachanova V, Torkelson C, Geller M, Haddad T, Shanley R, Kreitzer M. Mindfulness-based cancer recovery in survivors recovering from chemotherapy and radiation. Journal of Community and Supportive Oncology. 2015;14(8):351-8.

42. Chambers SK, Occhipinti S, Foley E, Clutton S, Legg M, Berry M, Stockler MR, Frydenberg M, Gardiner RA, Lepore SJ, Davis ID, Smith DP. Mindfulness-Based Cognitive Therapy in Advanced Prostate Cancer: A Randomized Controlled Trial. Journal of Clinical Oncology. 201701 20;35(3):291-297. https://doi.org/10.1200/jco.2016.68.8788

43. Henderson VP, Massion AO, Clemow L, Hurley TG, Druker S, Hébert JR. A Randomized Controlled Trial of 
Mindfulness-Based Stress Reduction for Women With EarlyStage Breast Cancer Receiving Radiotherapy. Integrative Cancer Therapies. 201301 28;12(5):404-413. https://doi. org/10.1177/1534735412473640

44. Hoffman CJ, Ersser SJ, Hopkinson JB, Nicholls PG, Harrington JE, Thomas PW. Effectiveness of MindfulnessBased Stress Reduction in Mood, Breast- and EndocrineRelated Quality of Life, and Well-Being in Stage 0 to III Breast Cancer: A Randomized, Controlled Trial. Journal of Clinical Oncology. 201204 20;30(12):1335-1342. https:// doi.org/10.1200/jco.2010.34.0331

45. Zhang R, Yin J, Zhou Y. Effects of mindfulness-based psychological care on mood and sleep of leukemia patients in chemotherapy. International Journal of Nursing Sciences. 2017 Oct;4(4):357-361. https://doi.org/10.1016/j. ijnss.2017.07.001

46. Liu T, Zhang W, Xiao S, Xu L, Wen Q, Bai L, Ma Q, Ji B. Mindfulness-based stress reduction in patients with differentiated thyroid cancer receiving radioactive iodine therapy: a randomized controlled trial. Cancer Management and Research. 2019 01;Volume 11:467-474. https://doi. org/10.2147/cmar.s183299

47. Nissen ER, O'Connor M, Kaldo V, Højris I, Borre M, Zachariae R, Mehlsen M. Internet-delivered mindfulnessbased cognitive therapy for anxiety and depression in cancer survivors: A randomized controlled trial. Psycho-Oncology. 2019 Nov;29(1):68-75. https://doi.org/10.1002/pon.5237

48. Kenne Sarenmalm E, Mårtensson LB, Andersson BA, Karlsson P, Bergh I. Mindfulness and its efficacy for psychological and biological responses in women with breast cancer. Cancer Medicine. 201704 18;6(5):1108-1122. https://doi.org/10.1002/cam4.1052

49. Compen F, Bisseling E, Schellekens M, Donders R, Carlson L, van der Lee M, Speckens A. Face-to-Face and Internet-Based Mindfulness-Based Cognitive Therapy Compared With Treatment as Usual in Reducing Psychological Distress in Patients With Cancer: A Multicenter Randomized Controlled Trial. Journal of Clinical Oncology. 201808 10;36(23):24132421. https://doi.org/10.1200/jco.2017.76.5669

50. Kingston T, Collier S, Hevey D, McCormick MM, Besani C, Cooney J, O'Dwyer AM. Mindfulness-based cognitive therapy for psycho-oncology patients: an exploratory study. Irish Journal of Psychological Medicine. 201501 07;32(3):265-274. https://doi.org/10.1017/ipm.2014.81

51. Lengacher CA, Reich RR, Paterson CL, Ramesar S, Park JY, Alinat C, Johnson-Mallard V, Moscoso M, BudhraniShani P, Miladinovic B, Jacobsen PB, Cox CE, Goodman M, Kip KE. Examination of Broad Symptom Improvement Resulting From Mindfulness-Based Stress Reduction in Breast Cancer Survivors: A Randomized Controlled Trial. Journal of Clinical Oncology. 201608 20;34(24):2827-2834. https://doi.org/10.1200/jco.2015.65.7874

52. Reich RR, Lengacher CA, Alinat CB, Kip KE, Paterson C, Ramesar S, Han HS, Ismail-Khan R, Johnson-Mallard V, Moscoso M, Budhrani-Shani P, Shivers S, Cox CE, Goodman M, Park J. Mindfulness-Based Stress Reduction in Posttreatment Breast Cancer Patients: Immediate and Sustained Effects Across Multiple Symptom Clusters. Journal of Pain and Symptom Management. 2017 01;53(1):85-95. https:// doi.org/10.1016/j.jpainsymman.2016.08.005

53. Bränström R, Kvillemo P, Moskowitz JT. A Randomized Study of the Effects of Mindfulness Training on Psychological Well-being and Symptoms of Stress in Patients Treated for Cancer at 6-month Follow-up. International Journal of Behavioral Medicine. 201109 20;19(4):535-542. https:// doi.org/10.1007/s12529-011-9192-3

54. Lengacher CA, Shelton MM, Reich RR, Barta MK, Johnson-
Mallard V, Moscoso MS, Paterson C, Ramesar S, Budhrani P, Carranza I, Lucas J, Jacobsen PB, Goodman MJ, Kip KE. Mindfulness based stress reduction (MBSR(BC)) in breast cancer: evaluating fear of recurrence (FOR) as a mediator of psychological and physical symptoms in a randomized control trial (RCT). Journal of Behavioral Medicine. 2014 Nov 27;37(2):185-195. https://doi.org/10.1007/s10865012-9473-6

55. Johns SA, Brown LF, Beck-Coon K, Talib TL, Monahan PO, Giesler RB, Tong Y, Wilhelm L, Carpenter JS, Von Ah D, Wagner CD, de Groot M, Schmidt K, Monceski D, Danh M, Alyea JM, Miller KD, Kroenke K. Randomized controlled pilot trial of mindfulness-based stress reduction compared to psychoeducational support for persistently fatigued breast and colorectal cancer survivors. Supportive Care in Cancer. 201605 17;24(10):4085-4096. https://doi. org/10.1007/s00520-016-3220-4

56. Johannsen M, O'Toole M, O'Connor M, Jensen A, Zachariae R. Clinical and psychological moderators of the effect of mindfulness-based cognitive therapy on persistent pain in women treated for primary breast cancer - explorative analyses from a randomized controlled trial. Acta Oncologica. 201701 17;56(2):321-328. https://doi.or g/10.1080/0284186x.2016.1268713

57. Johns SA, Brown LF, Beck-Coon K, Monahan PO, Tong Y, Kroenke K. Randomized controlled pilot study of mindfulness-based stress reduction for persistently fatigued cancer survivors. Psycho-Oncology. 201508 17;24(8):885893. https://doi.org/10.1002/pon.3648

58. Ledesma D, Kumano H. Mindfulness-based stress reduction and cancer: a meta-analysis. Psycho-Oncology. 2008 Nov 20;18(6):571-579. https://doi.org/10.1002/pon.1400

59. Barlow D, Allen L, Choate M. Towards a unified treatment for emotional disorders. Behavior Therapy. 2004;35:205-30.

60. Gotink RA, Chu P, Busschbach JJV, Benson H, Fricchione GL, Hunink MGM. Standardised Mindfulness-Based Interventions in Healthcare: An Overview of Systematic Reviews and Meta-Analyses of RCTs. Veves A. PLOS ONE. 201504 16;10(4):e0124344. https://doi.org/10.1371/journal. pone. 0124344



This work is licensed under a Creative Commons AttributionNon Commercial 4.0 International License. 